\title{
Highly collimated electron beams from double-gate field emitter arrays with large collimation gate apertures
}

\author{
P. Helfenstein, ${ }^{1, a)}$ E. Kirk, ${ }^{1}$ K. Jefimovs, ${ }^{2}$ T. Vogel, ${ }^{1}$ C. Escher, ${ }^{3}$ H.-W. Fink, ${ }^{3}$ and \\ S. Tsujino 1, b) \\ ${ }^{1}$ Laboratory for Micro- and Nanotechnology, Paul Scherrer Institut, CH-5232 Villigen-PSI, Switzerland \\ ${ }^{2}$ Electronics/Metrology/Reliability Laboratory, EMPA, Überlandstrasse 129, CH-8600 Dübendorf, \\ Switzerland \\ ${ }^{3}$ Physik Institut, University of Zurich, Winterthurerstrasse 190, CH-8057 Zurich, Switzerland
}

(Received 4 November 2010; accepted 12 January 2011; published online 9 February 2011)

\begin{abstract}
Electron collimation in field emitter arrays with electron extraction gate and collimation gate electrodes is studied with the goal to develop a high-brightness high current cathode. Using metallic field emitter arrays prepared by the molding method, we fabricated a stacked double-gate device with the two gates differing in diameter by a process utilizing focused-ion beam milling. We measured the field-emission beam characteristics and demonstrated a reduction of the emission angle by a factor of $7.1 \pm 0.8$ with minimal emission current decrease under collimating conditions, resulting in a current density increase by a factor of 13.9 \pm 1.0 . () 2011 American Institute of Physics. [doi:10.1063/1.3551541]
\end{abstract}

Double-gate field emitter arrays (FEAs) have been studied for high-brightness cathode applications. ${ }^{1-4}$ Microwave vacuum electronic devices using single-gate FEAs, which allow for a compact and simplified gun design, have been successfully demonstrated. ${ }^{5-8}$ However, a high current density field-emission electron beam with reduced transverse electron velocity spread is a crucial factor in extending the FEA-based vacuum electronic device technology to higher power densities and frequencies in the terahertz gap., ${ }^{9,10}$

In double-gate FEAs, microfabricated field emitters are equipped with an electron extraction gate $G_{\text {ext }}$ and a collimation gate $G_{\mathrm{col}}$. By applying a negative bias to $G_{\mathrm{col}}$, the fieldemission electron beam can be collimated. ${ }^{11-19}$ The reported structures differ in terms of location of $G_{\mathrm{col}}$ with respect to $G_{\text {ext }}$, as well as in the number of emitters per single $G_{\text {col }}$ aperture. Among these, stacked double-gate devices providing a $G_{\mathrm{col}}$ aperture for individual emitters exhibit the smallest electron beam emission angle. Toma et al. ${ }^{17}$ reported a factor of 15 beam size reduction, but it was accompanied by the decrease of the emission current by a factor of $\sim 10^{3}$. With volcano-structured double-gated FEAs, electrostatic shielding of the emitter tip from $G_{\mathrm{col}}$ in a nonplanar configuration largely prevented current reduction. ${ }^{19}$ Although this approach is promising for specific applications such as miniature electron guns for lithography or field-emission displays, device structures with a planar top surface are desirable for applications with high acceleration field strengths such as free electron lasers. ${ }^{2}$

In this letter, we report the fabrication and characterization by field-emission microscope (FEM) of double-gate FEAs with a planar top surface and different gate aperture diameters, which can minimize current reduction at high collimation voltages. ${ }^{4}$ By developing a focused-ion beam (FIB)assisted gate fabrication process and applying it to our molded molybdenum FEAs, we achieve a large reduction in

\footnotetext{
${ }^{\text {a) }}$ Author to whom correspondence should be addressed. Electronic mail: patrick.helfenstein@psi.ch.

${ }^{\mathrm{b}}$ Electronic mail: soichiro.tsujino@psi.ch.
}

emission angle accompanied by an increased current density.

Our molybdenum FEAs were fabricated by the molding method using silicon (001) wafer substrates. ${ }^{20}$ The molybdenum emitters with tip apex diameters of 10-20 nm have a square base with a side length of $\sim 1.5 \mu \mathrm{m}$ and a height of $\sim 1.2 \mu \mathrm{m}$. The emitter arrays are supported by $\sim 400 \mu \mathrm{m}$ of electroplated nickel. The $G_{\text {ext }}$ apertures were fabricated by a self-aligned etch-back and wet-etching process, ${ }^{20}$ which leads to square apertures with rounded corners. The achievable extraction gate radii-characterized by the distance from the tip to the closest point of $G_{\text {ext }}$-lie in the range of $1.8-2.5 \mu \mathrm{m}$. For the fabrication of the $G_{\mathrm{col}}$ apertures, we developed an FIB-assisted process. These were fabricated by FIB milling of a sacrificial mask layer and subsequent wetetching of the molybdenum $G_{\text {col }}$ layer. This enables us to flexibly adjust the $G_{\mathrm{col}}$ aperture size and shape. Figure 1 shows scanning electron microscope (SEM) micrographs of the fabricated device. In the experiment described below, we used $2 \times 2$ arrays with $10 \mu \mathrm{m}$ separation between tips and $G_{\text {ext }}$ and $G_{\text {col }}$ aperture radii of $1.15 \pm 0.05$ and $3.11 \pm 0.05 \mu \mathrm{m}$, respectively. After gate aperture fabrication, the $G_{\mathrm{col}}$ electrode was patterned into a $1.2 \mathrm{~mm}$ diameter circular shape.

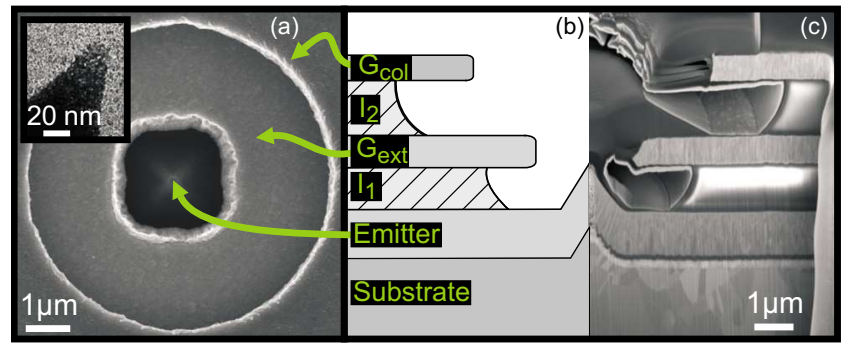

FIG. 1. (Color online) SEM micrographs of a double-gate FEA. (a) Top view and (c) cross-section. (b) Schematic diagram corresponding to the cross-section shown in (c). The shaded layers $\left(I_{1}\right.$ and $\left.I_{2}\right)$ are the $1.2 \pm 0.1 \mu \mathrm{m}$ thick $\mathrm{SiO}_{2}$ insulators. $G_{\text {ext }}$ and $G_{\text {col }}$ diameters are $2.30 \pm 0.05$ and $6.22 \pm 0.05 \mu \mathrm{m}$, respectively. The inlay in (a) shows a transmission electron microscope (TEM) image of the emitter tip apex. 


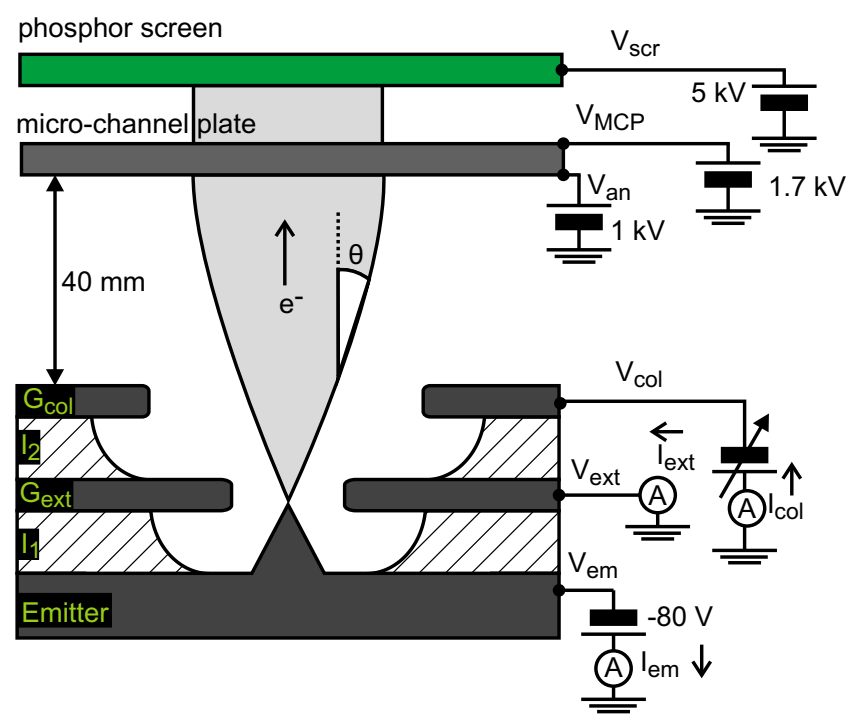

FIG. 2. (Color online) Schematic diagram of the experimental setup. The extracted electron beam is amplified by a microchannel plate biased to 0.7 $\mathrm{kV}$ given by $\left(V_{\mathrm{MCP}}-V_{\mathrm{an}}\right)$ and detected by the phosphor screen biased at a voltage $V_{\text {scr }}$ of $5 \mathrm{kV}$. The currents $I_{\mathrm{em}}, I_{\mathrm{ext}}$, and $I_{\mathrm{col}}$ were simultaneously recorded. $\theta$ denotes the emission angle of the electron beam at the emitter surface.

The schematic setup of the FEM experiment is depicted in Fig. 2. The FEA chip was mounted on a polyether ether ketone holder and inserted into the FEM chamber which was evacuated to $\sim 5 \times 10^{-8}$ mbar. The gate electrodes of up to three double-gate FEAs were wire bonded and connected to electrical feedthroughs. Field emission was initiated by applying negative voltage $V_{\mathrm{em}}$ to the emitters while connecting $G_{\text {ext }}$ to ground potential. The extracted electron beam was collimated by applying negative voltage $V_{\text {col }}$ to $G_{\text {col }}$. We placed the FEA chip at a distance of $40 \mathrm{~mm}$ to the microchannel plate (MCP) and the phosphor screen. The emitted electrons were accelerated by a voltage $V_{\text {an }}$ of $1 \mathrm{kV}$ applied to the MCP entrance plate, amplified by the MCP, and imaged on the phosphor screen. During the experiment, the current $I_{\mathrm{em}}$ injected into the emitter substrate, the current $I_{\mathrm{ext}}$ flowing through $G_{\text {ext }}$, and the current $I_{\text {col }}$ intercepted by $G_{\mathrm{col}}$ were recorded simultaneously with the electron beam image. The net emission current $I_{\text {net }}$ that reached the screen assembly was evaluated as $\left(\left|I_{\mathrm{em}}\right|-I_{\text {ext }}-I_{\mathrm{col}}\right)$. At first, the FEA chip was conditioned by scanning $V_{\text {em }}$ between 0 and $-160 \mathrm{~V}$ until $I_{\text {net }}$ reached a few microamperes and the current-voltage characteristics stabilized. Subsequently, we applied a constant $V_{\text {em }}$ of $-80 \mathrm{~V}$ with $I_{\text {net }}$ between 0.5 and $1 \mathrm{nA}$ to avoid destructive arcing.

In Fig. 3, we show three electron beam images which display the measured reduction in electron beam size and an increase in current density for $V_{\text {col }}$ equal to $+1,-31$, and
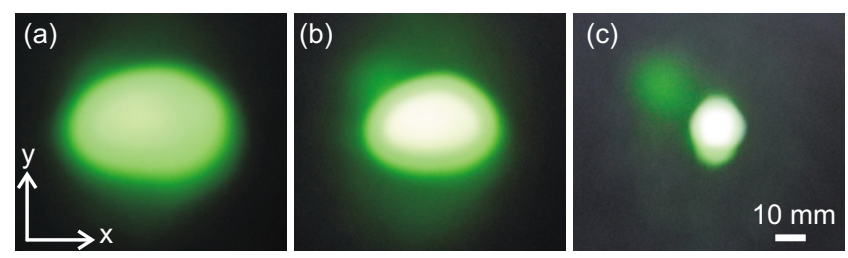

FIG. 3. (Color online) Field-emission microscope images of electron beam at constant extraction bias $V_{\text {em }}$ of $-80 \mathrm{~V}$ at collimation voltages $V_{\text {col }}$ of (a) +1 , (b) -31 , and (c) $-61 \mathrm{~V}$.

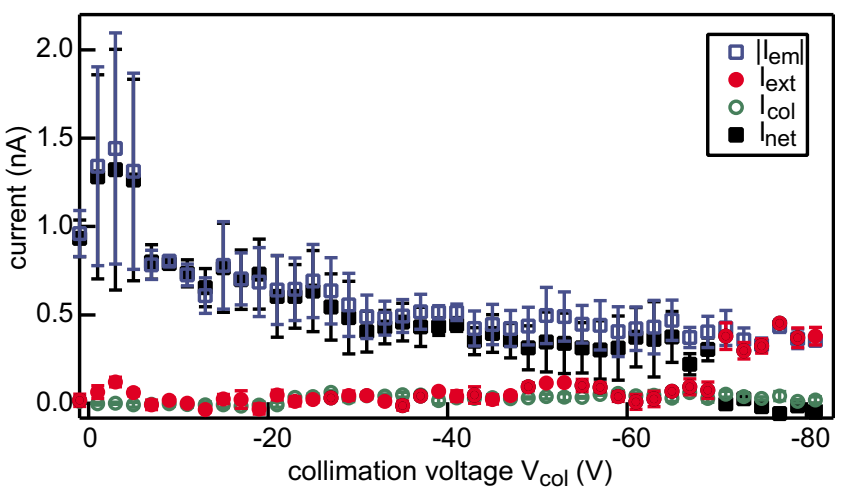

FIG. 4. (Color online) Absolute value of emitter current $I_{\mathrm{em}}$ (open squares), net current $I_{\text {net }}$ (filled squares), extraction gate current $I_{\text {ext }}$ (filled circles), and the collimation gate current $I_{\text {col }}$ (open circles) at various $V_{\text {col }}$ 's for $V_{\text {em }}$ fixed at $-80 \mathrm{~V}$.

$-61 \mathrm{~V}$, whereas $V_{\mathrm{em}}$ was fixed at $-80 \mathrm{~V}$. The reduction in electron beam size and the increasing beam brightness with the decrease of $V_{\text {col }}$ indicate that the decrease of $I_{\text {net }}$ with the decrease of $V_{\mathrm{col}}$ is minimal in this voltage range. This is shown by the simultaneously measured current summarized in Fig. 4 for a fixed $V_{\mathrm{em}}$ of $-80 \mathrm{~V}$. $I_{\text {net }}$ was equal to $0.5 \pm 0.2 \mathrm{nA}$ for $V_{\text {col }}$ between +1 and $-65 \mathrm{~V}$, whereas $I_{\text {ext }}$ and $I_{\text {col }}$ were equal to $0.04 \pm 0.03 \mathrm{nA}$ and therefore below $\sim 20 \%$ of $I_{\text {net }}$. In Fig. 5(a) we show the variation of the current density on the phosphor screen with the decrease of $V_{\text {col}}$. The current density was evaluated by dividing $I_{\text {net }}$ by the beam size for each $V_{\text {col }}$. We found that when $V_{\text {col }}$ was decreased from 0 to $-69 \mathrm{~V}$, the current density increased by a factor of $13.9 \pm 1.0$. We note that $I_{\text {net }}$ falls to zero for $V_{\text {col }}$ beyond $-69 \mathrm{~V}$ since the electrons are repelled by the potential field of $G_{\mathrm{col}}$ and collected by $G_{\text {ext }}$ similarly to previous reports. $^{8-16}$

The increased current density at maximal collimation is due to the reduction of the emission angle $\theta$ depicted in Fig.
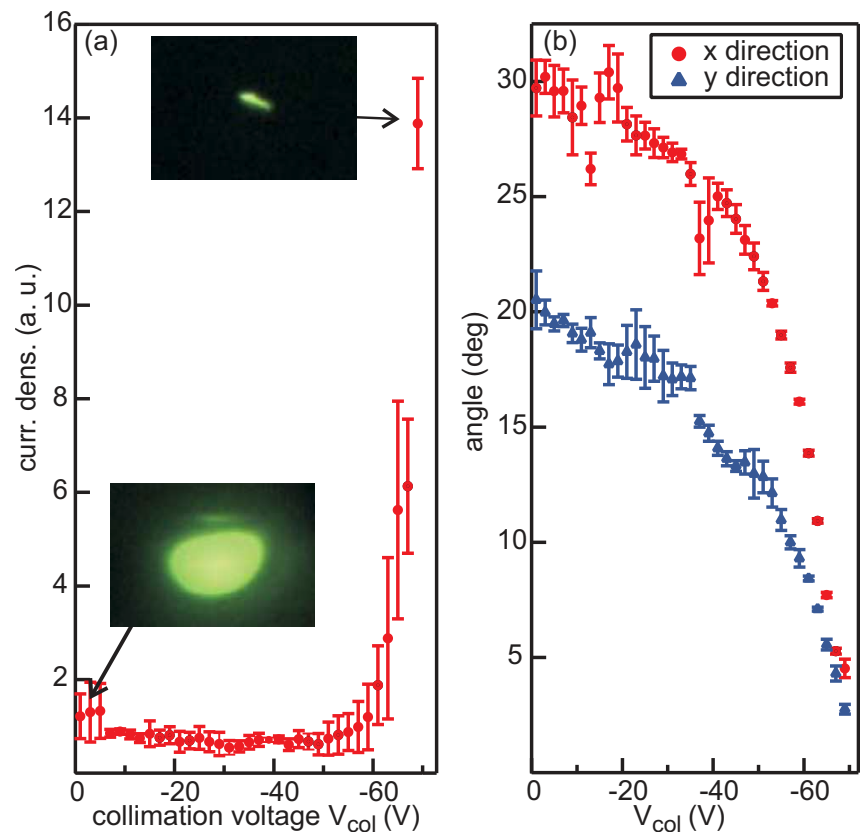

FIG. 5. (Color online) (a) Current density measured on the phosphor screen plotted against $V_{\text {col }}$ for fixed $V_{\text {em }}$. The two inlaid pictures show the electron beam at $V_{\text {col }}$ of $0 \mathrm{~V}$ (bottom) and $-69 \mathrm{~V}$ (top). (b) The relation between the emission angle $\theta$ and $V_{\text {col }}$ with the directions as defined in Fig. 3. 
2. It is defined as the half width spread of the field-emission electron beam. Using Eq. (1), we evaluated $\theta$ at each $V_{\text {col }}$ as presented in Fig. 5(b),

$$
\frac{D}{L}=\frac{2 \tan \theta}{1+\sqrt{1+\frac{V_{\mathrm{an}}}{\left|V_{\mathrm{em}}\right| \cos ^{2} \theta}}} .
$$

Herein, the electron beam radius $D$ was obtained from the half width of the half maximum intensity of the phosphor screen image, and $L$ is the distance from the FEA to the MCP and phosphor screen. We found that $\theta$ in the x-direction decreased from $29.7^{\circ} \pm 1.2^{\circ}$ to $4.5^{\circ} \pm 0.4^{\circ}$ when $V_{\text {col }}$ was decreased from 0 to $-69 \mathrm{~V}$. $\theta$ in the y-direction decreased from $20.5^{\circ} \pm 1.2^{\circ}$ to $2.7^{\circ} \pm 0.2^{\circ}$ at the same time. The reduction factor of $\theta$ amounts to $(6.6 \pm 0.8)$ and $(7.6 \pm 0.8)$ in $x-$ and $y$-directions, respectively. The asymmetry of the electron beam shape is ascribed to the residual potential distribution of the setup such as the gate contact wires and is partly compensated by the acceleration potential $V_{\text {an }}$.

In summary, using all-metal FEAs combined with selfaligned and FIB-assisted processes, we demonstrated a 14fold current density increase and a minimal current loss to the extraction gate at large negative collimation voltages. We achieved this by increasing the $G_{\text {col }}$ aperture diameter to $6.2 \mu \mathrm{m}$ through the application of our FIB-assisted process. We thus attained a larger ratio between the two gate-to-tip distances-from emitter tip apex to $G_{\text {col }}$ edge and from emitter tip apex to $G_{\text {ext }}$ edge-than would have been achievable with the self-aligned process. Also, this larger ratio accounts for the partial shielding of $G_{\mathrm{col}}$ from the emitter tip by $G_{\text {ext. }}{ }^{19}$ Our FIB-assisted process was utilized to produce arrays with well-defined geometric shapes. Further investigations into high tip current operation and upscaling of the emitter array size are needed to realize a high-brightness and high current cathode. Additionally, we expect further improvement in the beam brightness from optimization of the device structure in terms of the gate aperture sizes and the thicknesses of the gate and insulator layers.
The authors would like to thank J. Krbanjevic and E. Minikus for preparing the TEM lamella and taking TEM micrographs. This work was partially supported by the SwissFEL project of the Paul Scherrer Institute and the Swiss National Science Foundation.

${ }^{1}$ M. Dehler, A. Candel, and E. Gjonaj, J. Vac. Sci. Technol. B 24, 892 (2006).

${ }^{2}$ B. D. Patterson, R. Abela, H. H. Braun, U. Flechsig, R. Ganter, Y. Kim, E. Kirk, A. Oppelt, M. Pedrozzi, S. Reiche, L. Rivkin, T. Schmidt, B. Schmitt, V. N. Strocov, S. Tsujino, and A. F. Wrulich, New J. Phys. 12, 035012 (2010).

${ }^{3}$ C. M. Tang, M. Goldstein, T. A. Swyden, and J. E. Walsh, Nucl. Instrum. Methods Phys. Res. A 358, 7 (1995).

${ }^{4}$ S. Tsujino, P. Helfenstein, E. Kirk, T. Vogel, C. Escher, and H. W. Fink, IEEE Electron Device Lett. 31, 1059 (2010).

${ }^{5}$ H. Makishima, S. Miyano, H. Imura, J. Matsuoka, H. Takemura, and A. Okamoto, Appl. Surf. Sci. 146, 230 (1999).

${ }^{6}$ D. R. Whaley, B. M. Gannon, V. O. Heinen, K. E. Kreischer, C. E. Holland, and C. A. Spindt, IEEE Trans. Plasma Sci. 30, 998 (2002).

${ }^{7}$ D. R. Whaley, B. M. Gannon, C. R. Smith, C. M. Armstrong, and C. A. Spindt, IEEE Trans. Plasma Sci. 28, 727 (2000).

${ }^{8}$ D. R. Whaley, R. Duggal, C. M. Armstrong, C. L. Bellew, C. E. Holland, and C. A. Spindt, IEEE Trans. Electron Devices 56, 896 (2009).

${ }^{9}$ J. H. Booske, Phys. Plasmas 15, 055502 (2008).

${ }^{10}$ R. L. Ives, IEEE Trans. Plasma Sci. 32, 1277 (2004).

${ }^{11}$ L. Dvorson, G. Sha, I. Kymissis, C. Y. Hong, and A. I. Akinwande, IEEE Trans. Electron Devices 50, 2548 (2003).

${ }^{12}$ A. Hosono, S. Kawabuchi, S. Horibata, S. Okuda, H. Harada, and M. Takai, J. Vac. Sci. Technol. B 17, 575 (1999).

${ }^{13}$ J. Itoh, Y. Toma, K. Morikawa, S. Kanemaru, and K. Shimizu, J. Vac. Sci. Technol. B 13, 1968 (1995).

${ }^{14}$ C. Py, M. Gao, S. R. Das, P. Grant, P. Marshall, and L. LeBrun, J. Vac. Sci. Technol. A 18, 626 (2000).

${ }^{15} \mathrm{C}$. Py, J. Itoh, T. Hirano, and S. Kanemaru, IEEE Trans. Electron Devices 44, 498 (1997).

${ }^{16}$ Y. Yamaoka, S. Kanemaru, and J. Itoh, Jpn. J. Appl. Phys., Part 1 35, 6626 (1996).

${ }^{17}$ Y. Toma, S. Kanemaru, and J. Itoh, J. Vac. Sci. Technol. B 14, 1902 (1996).

${ }^{18}$ M. Nagao, T. Yoshida, S. Kanemaru, Y. Neo, and H. Mimura, Jpn. J. Appl. Phys. 48, 06FK02 (2009).

${ }^{19}$ Y. Neo, M. Takeda, T. Soda, M. Nagao, T. Yoshida, S. Kanemaru, T. Sakai, K. Hagiwara, N. Saito, T. Aoki, and H. Mimura, J. Vac. Sci. Technol. B 27, 701 (2009).

${ }^{20}$ E. Kirk, S. Tsujino, T. Vogel, K. Jefimovs, J. Gobrecht, and A. Wrulich, J. Vac. Sci. Technol. B 27, 1813 (2009). 